\title{
El portafolio de evidencias como instrumento metacognitivo en el proceso de titulación de estudiantes normalistas
}

\section{The evidence portfolio as a metacognitive instrument in the normalist student graduation process}

DOI: $10.46932 /$ sfjdv3n1-077

Received in: Jan 30st, 2021

Accepted in: Feb 1th, 2022

\author{
Carlos Alfredo Damián García \\ Dr. En Investigación Educativa \\ Esc. Normal Experimental \\ "Salvador Varela Reséndiz" \\ Av.de la Normal s/n Juchipila, Zac. México \\ E-mail: cabritoreposado@gmail.com
}

\begin{abstract}
RESUMEN
El presente trabajo corresponde al reporte final de una investigación longitudinal de 3 años, para conocer cómo influye el uso del portafolio de evidencias como instrumento metacognitivo en el proceso de titulación de los estudiantes del octavo semestre de la Licenciatura en Educación Primaria de la Escuela Normal de Juchipila, Zac. Participaron 1 docente asesor, 3 maestros titulares de educación primaria y 3 estudiantes asesorados. Estos normalistas realizaron procesos de planeación, actuación, observación, y reflexión de secuencias didácticas aplicadas en su práctica docente, vinculadas a las competencias genéricas y profesionales que seleccionaron para disertar su documento de titulación a través del portafolio de evidencias. El método que se utilizó fue la investigación-acción, con las técnicas de observación y el modelo de Kemmis (1989) a través de instrumentos de tipo cualitativo como notas de campo, ficha de autocorrección, rúbrica, fotografía, videograbación, entrevista y matriz de consistencia. Los resultados aportan que la mayoría de las reflexiones registradas en los portafolios de evidencias, corresponden al nivel de reflexión crítica (Lamas, 2016, como se citó en Rodrigues, 2013) propiciado por un acompañamiento metacognitivo, evidenciando una mejora continua en las competencias profesionales de los alumnos.
\end{abstract}

Palabras clave: portafolio de evidencias, metacognición, secuencia didáctica, matriz de consistencia.

\begin{abstract}
The present work corresponds to the final report of a 3-year longitudinal investigation, to know how the use of the evidence portfolio as a metacognitive instrument influences the qualification process of the students of the eighth semester of the Bachelor of Primary Education of the Normal School of Juchipila, Zac. 1 advisory teacher, 3 tenured teachers of primary education and 3 advised students participated. These normalistas carried out processes of planning, acting, observing, and reflecting on the didactic sequences applied in their teaching practice, linked to the generic and professional skills they selected to dissert their degree document through the portfolio of evidence. The method used was action research, with the observation techniques and the Kemmis (1989) model through qualitative instruments such as field notes, self-correction sheet, rubric, photography, video recording, interview and matrix of consistency. The results provide that most of the reflections registered in the evidence portfolios correspond to the level of critical reflection (Lamas, 2016, as cited in Rodrigues, 2013) fostered by a
\end{abstract}


metacognitive accompaniment, evidencing a continuous improvement in the professional competencies of the students.

Keywords: evidence portfolio, metacognition, didactic sequence, consistency matrix.

\section{INTRODUCCIÓN}

El presente estudio longitudinal inició en el año 2018 donde participaron 3 docentes y 23 estudiantes de la Licenciatura en Educación primaria de la Escuela Normal Experimental "Salvador Varela Reséndiz" de Juchipila, Zac. En el primer reporte de investigación, se mencionó la importancia de implementar el portafolio electrónico como instrumento metacognitivo en la formación inicial del profesorado, donde los estudiantes lograron ser más conscientes en el diseño, aplicación y evaluación de sus estrategias de aprendizaje en sus jornadas de práctica docente, sistematizando la información en el primer nivel de reflexión denominado como "técnico" (Lamas, 2016, citó en Rodrigues, 2013), donde también identificaron sus necesidades de aprendizaje, tanto en su formación en la Escuela Normal como en su formación en la Escuela Primaria.

El segundo reporte de investigación se realizó en el año 2019, el cual, no solo consistió en un portafolio digital, sino que se apoyó a los estudiantes en su práctica docente con rúbricas y trípticos tutoriales, seccionando la información en planeación, evidencias y evaluación. Favoreció el trabajo autónomo y la sistematización de la experiencia bajo el ciclo reflexivo de Smyth (1989), donde identificaron sus fortalezas y áreas de oportunidad, logrando categorizarlas y permitiendo hacer propuestas de mejora.

\section{DESARROLLO}

El presente trabajo reporta el final de la investigación aplicado en el año 2020, donde solo participaron un docente como asesor y 3 estudiantes (del mismo grupo longitudinal) como asesorados de su proceso de titulación, bajo la modalidad de portafolio de evidencias de la Licenciatura en Educación Primaria. Cada estudiante desarrolló su creatividad y tuvo libertad en la construcción de su portafolio de evidencias, pero orientado por el docente asesor, a través de una rúbrica para su estructura y otra para las características de su contenido. Además, entre todos los participantes, elaboraron una matriz de consistencia apoyada con fichas de autocorrección de contenido, facilitando de manera metacognitiva la sistematización, análisis y reflexión de las evidencias de sus competencias genéricas y profesionales, tanto en su trayecto de formación en la Escuela Normal, como en su trayecto de práctica profesional en las escuelas primarias. 
Dichas competencias se presentaron de manera deductiva, es decir, demostraron evidencias de todas las competencias en forma general hasta el $6^{\circ}$ semestre. En el $7^{\circ}$ semestre aplicaron una evaluación diagnóstica al grupo de educación primaria donde realizaron su práctica profesional y realizaron una autoevaluación de sus competencias genéricas y profesionales, identificando las siguientes áreas de oportunidad: la evaluación formativa en español, los ambientes de aprendizaje en matemáticas y la enseñanza de la formación cívica y ética. Por lo tanto, cada alumno trabajó una temática y seleccionó las competencias afines, que constan de las siguientes:

- Genera ambientes formativos para propiciar la autonomía y promover el desarrollo de las competencias en los alumnos de educación básica.

- Emplea la evaluación para intervenir en los diferentes ámbitos y momentos de la tarea educativa.

- Actúa de manera ética ante la diversidad de situaciones que se presentan en la práctica profesional.

\subsection{MARCO TEÓRICO}

A partir de lo anterior, se consideró el concepto de la SEP (2012) de Portafolio de evidencias que consiste en la elaboración de:

un documento que reconstruye el proceso de aprendizaje del estudiante a partir de un conjunto de evidencias reflexionadas, analizadas, evaluadas y organizadas según la relevancia, pertinencia y representatividad respecto a las competencias genéricas y profesionales, con la intención de dar cuenta del nivel de logro o desempeño del estudiante en el ámbito de la profesión docente. (p. 19)

En este proceso se requiere definir qué competencias se va a demostrar, se seleccionan las evidencias más significativas, se reflexiona y analiza dichas evidencias y se proyecta una valoración de sí mismo, dando a conocer sus fortalezas y áreas de oportunidad.

En este sentido, dichos portafolios se elaboraron de manera metacognitiva, entendiéndose dicho término, conforme a la siguiente definición propia:

La metacognición es un proceso mental autoconsciente de la sensación, percepción, atención, memoria, cognición y actuación implicadas en una interacción cualquiera, con la intención de autorregular su aprendizaje a través de un análisis y reflexión de manera introspectiva, retrospectiva, prospectiva y crítica, demandando un pensamiento y desempeño estratégico.

Por lo tanto, encontramos una cierta relación con el Modelo Educativo para la Educación Obligatoria, donde se establece que:

La formación inicial debe desarrollar en los maestros la capacidad de aprender a aprender. Al igual que a los estudiantes, el vertiginoso avance en el conocimiento y el acelerado cambio social en el que vivimos obliga a los docentes a seguir aprendiendo a lo largo de sus vidas. (SEP, 2017, p. 142). 
Así mismo, es congruente con los dominios, criterios e indicadores del perfil docente (SEP, 2020) donde indica la importancia de organizar y adecuar el espacio escolar, tiempo, formas de organizar al grupo, materiales disponibles, actividades de autoevaluación y coevaluación para que los alumnos asuman la responsabilidad de su propio aprendizaje, la construcción conjunta de normas para garantizar una convivencia armónica y asertiva. Además, enfatiza en la reflexión de la práctica docente para mejorarla con base en las evidencias de los aprendizajes logrados por los alumnos.

Ante esto, es importante evitar una educación fragmentada, caracterizada por separar la planeación de la enseñanza para posteriormente evaluar el logro de sus objetivos. Transitar de un enfoque centrado en los contenidos a un enfoque centrado en las competencias, donde es importante partir de situaciones y problemas del contexto con el propósito de potenciar capacidades y saberes (conocer, hacer y ser) dentro de una perspectiva socio formativa. Así pues, para lograrlo, los jóvenes retomaron las secuencias didácticas, entendiéndolas como "conjuntos articulados de actividades de aprendizaje y evaluación que, con la mediación de un docente, buscan el logro de determinadas metas educativas, considerando una serie de recursos" (Tobón, Pimienta y García, 2010: 20).

Las secuencias didácticas fueron integradas en el trabajo de investigación acción con el modelo de Kemmis (1989), donde se sistematizó la información en cuatro fases interrelacionadas: planificación, acción, observación y reflexión. Cada uno de los momentos implicó una mirada retrospectiva, y una intención prospectiva que forman conjuntamente una espiral autorreflexiva de conocimiento y acción.

Todo este proceso de investigación acción fue construido durante el $7^{\circ}$ y $8^{\circ}$ semestre con el formato general de una matriz de consistencia.

La matriz de consistencia es:

un instrumento valioso que consta de un cuadro formado por columnas y filas; permite consolidar
los elementos clave de todo el proceso de investigación, además posibilita evaluar el grado de
coherencia y conexión lógica entre el título, el problema, la hipótesis, los objetivos, las variables,
el diseño de investigación seleccionado, los instrumentos de investigación, así como la población
y la muestra de estudio. (Abrigo 2018 cita a Carrasco, 2018)

Esta herramienta de trabajo coadyuva en la visualización, sistematización, verificación, análisis y reflexión de las evidencias. Además, se proporcionaron unas fichas de autocorrección de contenido para propiciar el nivel de reflexión crítica (Lamas, 2016, como se citó en Rodrigues, 2013):

Se refiere a la reflexión sobre las implicancias de las realidades sociales y políticas, así como de la moral y la ética, y las consecuencias de las prácticas en el aula, centrando su atención en el contexto y en las condiciones en las que se desarrollan. Se evidencia la actividad investigativa y la reflexión crítica de las acciones de enseñanza y sus consecuencias, así como sobre los procesos de pensamiento. 


\subsection{PLANTEAMIENTO DEL PROBLEMA}

A partir del análisis de los resultados del primer avance de investigación, donde se aplicó el Test de estilos de aprendizaje de Honey Alonso (1995), se identificó que en los 23 estudiantes predominaba el estilo pragmático, teórico y activo, resultando a la baja el estilo reflexivo. En el segundo reporte de investigación, la mayoría de los alumnos mejoraron sus intervenciones en el proceso reflexivo, pero a un nivel técnico según Van Manen (1977), donde analizan situaciones de manera aislada y superficial. Esto se vuelve a retomar, para propiciar una consolidación en las competencias genéricas y profesionales.

Por lo tanto, se establecieron las siguientes preguntas de investigación:

¿Cómo influye el uso del portafolio de evidencias como instrumento metacognitivo en el proceso de titulación de los estudiantes normalistas?

Se derivan las siguientes preguntas secundarias:

¿Cómo realizar un acompañamiento mediante el uso del portafolio de evidencias que elaboran de manera metacognitiva los estudiantes, desde el primer semestre hasta el octavo semestre de su formación inicial?

¿Qué efectos produce el uso de la secuencia didáctica y de la matriz de consistencia en la construcción del portafolio de evidencias?

Objetivo general de la investigación:

Identificar cómo influye el uso del portafolio de evidencias como instrumento metacognitivo en el proceso de titulación de los estudiantes normalistas.

Objetivos específicos:

- Implementar un acompañamiento mediante el uso del portafolio de evidencias que elaboran de manera metacognitiva los estudiantes, desde el primer semestre hasta el octavo semestre de su formación inicial.

- Conocer los efectos que produce el uso de la secuencia didáctica y de la matriz de consistencia en la construcción del portafolio de evidencias.

De estas reflexiones surge el siguiente supuesto:

El uso del portafolio de evidencias elaborado por los estudiantes como instrumento metacognitivo, mejora la planeación, actuación, observación y reflexión de sus competencias genéricas y profesionales, propiciando una autorregulación de su aprendizaje, viéndose motivado a la reflexión crítica durante el trayecto de su formación inicial. 


\section{MÉTODO}

El método que se utilizó fue la investigación-acción, con las técnicas de observación y el modelo de Kemmis (1989) a través de instrumentos de tipo cualitativo como notas de campo, ficha de autocorrección, rúbrica, fotografía, videograbación, entrevista y matriz de consistencia.

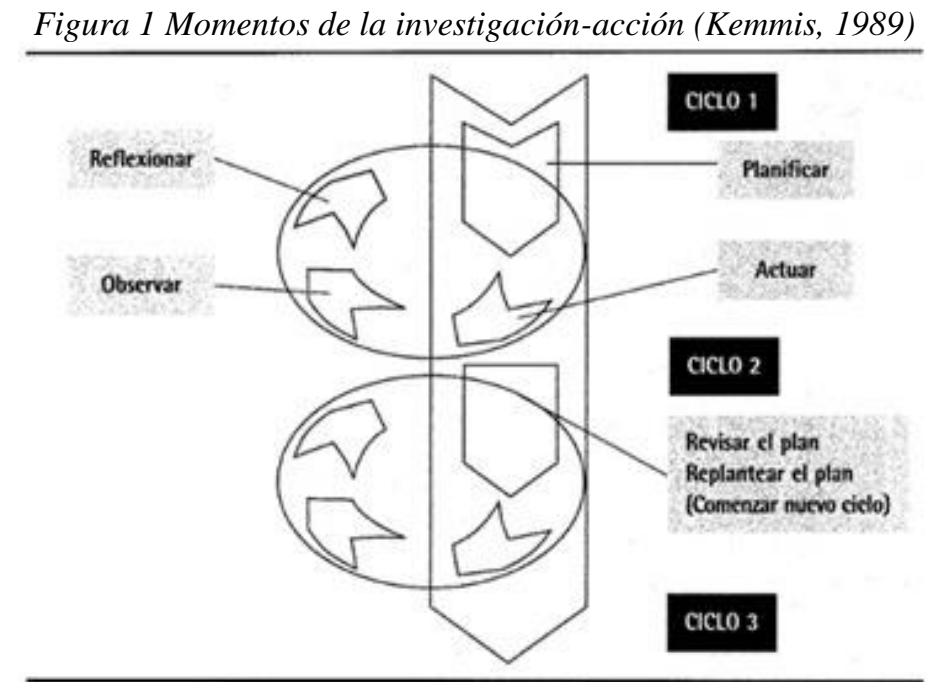

Nota. Retomado de La investigación acción, Antonio Latorre 2005.

\section{RESULTADOS}

Con la triangulación de datos y el apoyo de las fichas de autocorrección metacognitivas, los alumnos lograron con mayor autonomía, analizar sus datos por ciclos de investigación y presentarlos en gráficos, que al concentrar los resultados de los 3 asesorados, se deriva lo siguiente:

Figura 2 Tipos de evidencia por su contenido

\section{Tipos de evidencia}

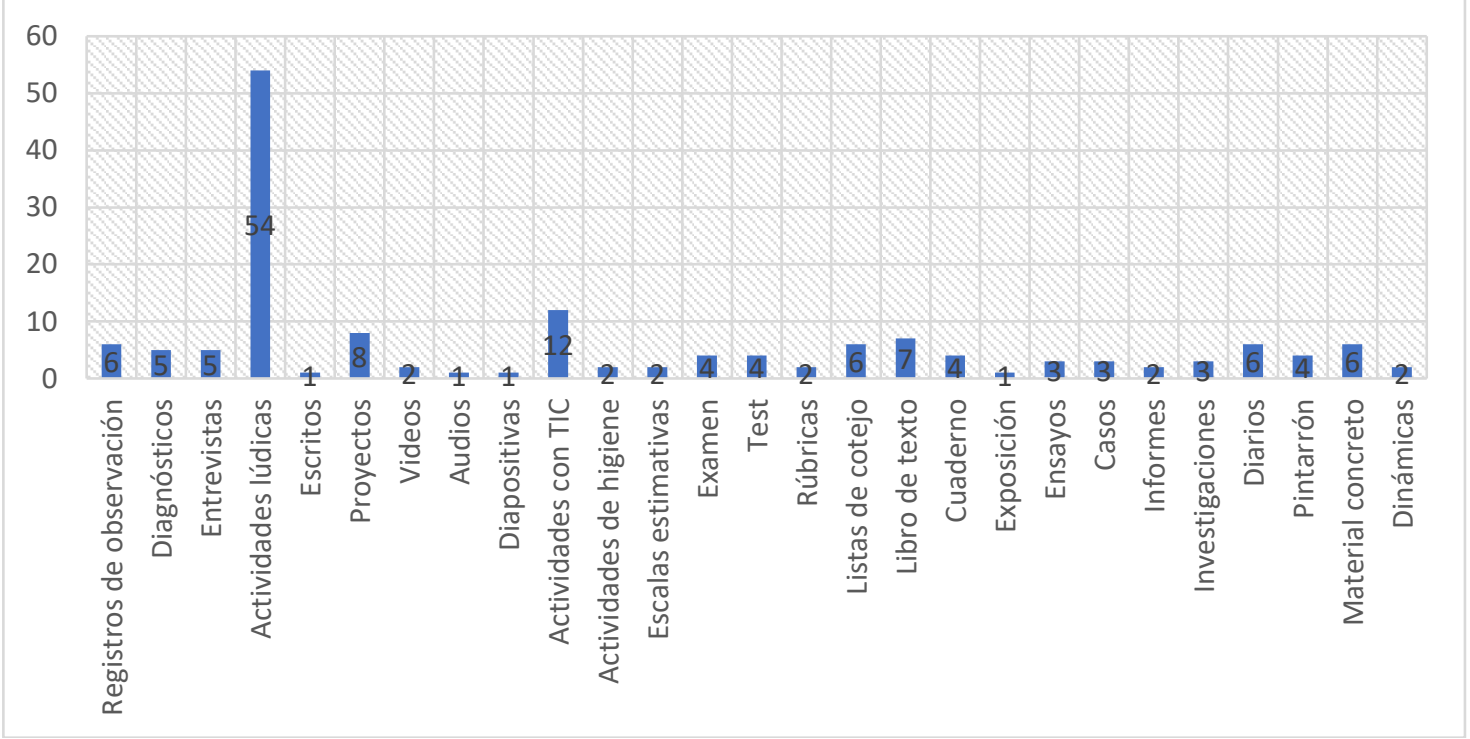


En la Figura 2, se identifica a las actividades lúdicas como las evidencias predominantes en el desarrollo de los portafolios y los videos, audios, diapositivas y exposiciones aparecen con menor frecuencia. Esto obedece a que, éstos estudiantes normalistas les gusta diseñar actividades con material concreto y que impliquen el juego, ya que su canal de aprendizaje predominante es el kinestésico. Sin embargo, insertaron muy pocas evidencias de actividades digitales, debido a que la mayoría de las Escuelas primarias donde practicaron, se localizan en medios rurales y semiurbanos, donde no existen o no funcionan los equipos de cómputo o de proyección.

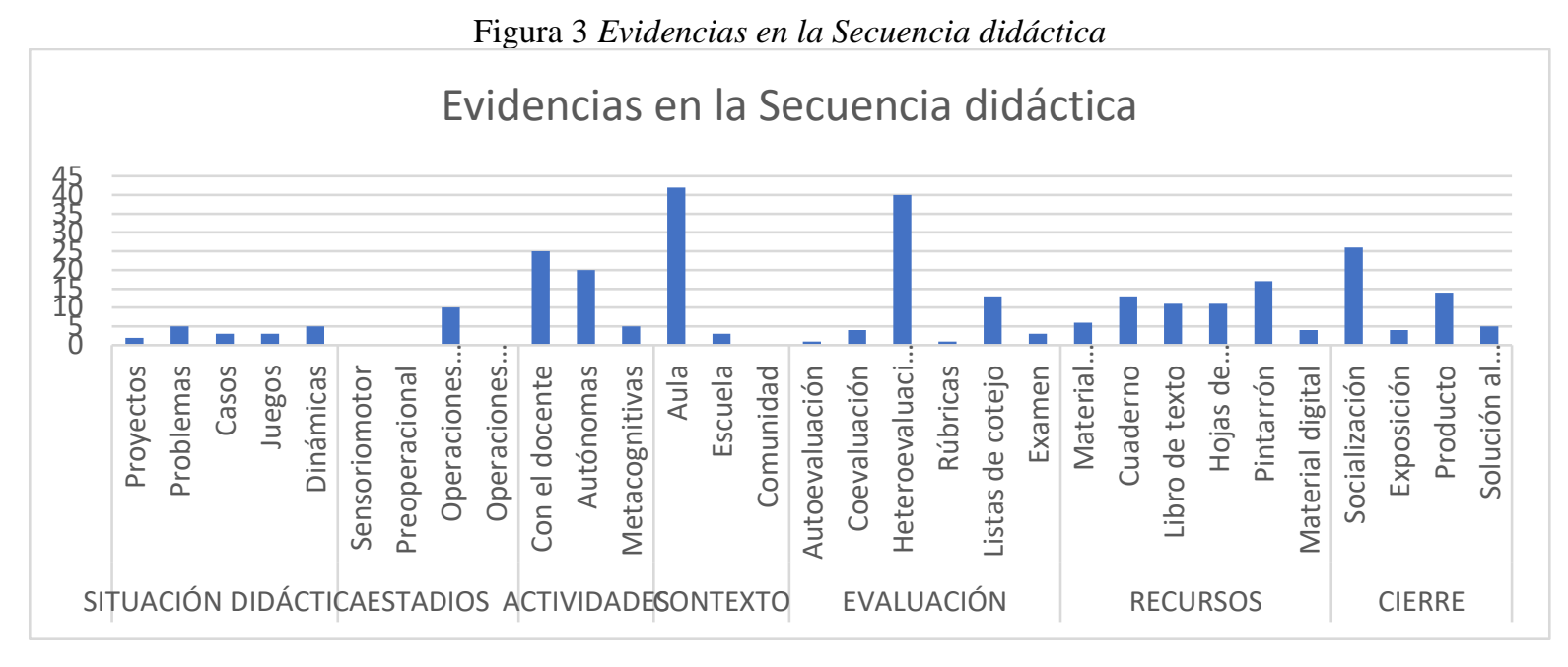

En la figura 3 podemos visualizar lo que se consideró como elementos básicos en un plan de clase, con modalidad de secuencia didáctica. De entrada, detectamos que los problemas y las dinámicas fueron las situaciones didácticas más utilizadas en clase, ubicadas en la etapa de operaciones concretas de acuerdo a Piaget. Así mismo, se aplicaron más actividades de aprendizaje en el aula y con el apoyo del docente, pues el trabajar fuera del aula se convertía en un reto, ya que la mayoría de las escuelas tienen un patio reducido que es más usado por el maestro de Educación Física. En otros casos, cuentan con un espacio amplio, pero sin domo, afectando el calor del sol el desarrollo de dichas actividades. En cuanto a la evaluación, en la mayoría de las secuencias de aprendizaje se heteroevaluó a los alumnos con listas de cotejo. Esta práctica evaluativa se manifestó con mayor uso debido a que los estudiantes normalistas conocen, pero no dominan el diseño de rúbricas u otros instrumentos de evaluación, teniendo inseguridad al querer aplicarlos. Los recursos más utilizados fueron el pintarrón y el cuaderno; lo menos utilizado fue el material digital. Por último, predominan las actividades de socialización en los momentos de cierre de una secuencia didáctica. 
Figura 4 Evidencias en la Matriz de consistencia

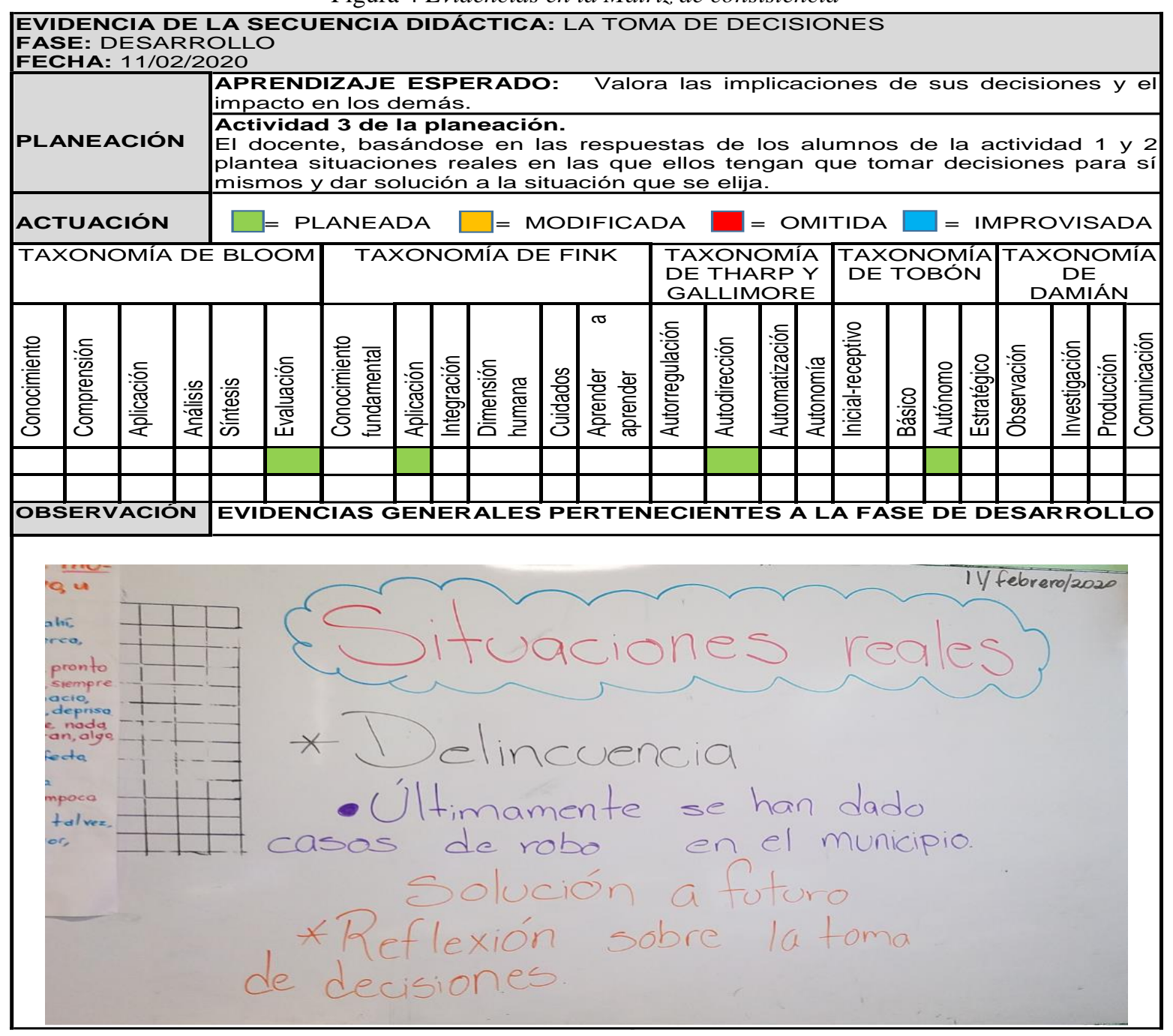

La figura 4 es un ejemplo de matriz de consistencia utilizado por los asesorados, convirtiéndose en una herramienta de apoyo, para concentrar la información conforme al modelo de Kemmis (1989), en 4 filas: lo que planificaron, lo que hicieron, lo que observaron de sus evidencias y lo que reflexionaron en la acción y sobre la acción de su práctica docente. La siguiente figura es continuación de la matriz. 
Figura 5 Reflexión sobre la acción REFLEXIÓN

Una vez analizadas las respuestas de las preguntas de la actividad uno, de manera general se dedujo que una de las principales problemáticas en el municipio es la delincuencia. Fueron los mismos alumnos quienes se encargaron de descubrir la situación, platicaron entre ellos, aportaron información que conocían sobre casos que se presentaron en el mismo pueblo. Yo como docente, simplemente me encargué de dar un ejemplo y ellos definieron y dieron la solución a la misma situación mediante una toma de decisiones reflexiva y llegaron a la siguiente conclusión sin que yo interviniera para su formulación.

-Erasmo: "Podemos decidir poner cámaras, tener perros bravos o poner cercas con electricidad, pero lo más importante es que nosotros somos los que podemos decidir si somos malos o somos buenos, así que a futuro si se podría ver una diferencia por la forma de tomar las decisiones nosotros mismos".

Realmente es una respuesta sorprendente, el grado de reflexión de los alumnos, en su mayoría ha llegado muy lejos, han conseguido darse cuenta claramente que mediante la toma de decisiones nosotros determinamos si hacemos el bien o el mal, es aquí cuando me doy cuenta que las actividades surten efecto y que realmente se mantiene al grupo interesado, enfocado y creando sus conocimientos para después llevarlos a la aplicación en su vida real.

Es admirable cuando niñas y niños de 10 y 11 años se relacionan directamente con la Formación Cívica y Ética. Su formulación de ideas, sus aportaciones claras que pudiesen para un adulto significar nada pero, para ellos pueden ser de carácter sumamente importante. La idea antes expresada nos deja un claro ejemplo de la madurez con la que puede llegar a contar un niño y esto se contrasta con el estadío de las operaciones concretas definido por Jean Piaget que va desde los 7 hasta los 11 años y nos dice que es cuando "el niño empieza a utilizar las operaciones mentales y la lógica para reflexionar sobre los hechos y los objetos de su ambiente" Linares, A. (2007 - 2008).

La evidencia se relaciona con la unidad 3 (asume los principios y reglas establecidas por la sociedad para la mejor convivencia) de la competencia genérica "Actúa con sentido ético". Su relación se debe principalmente a que el futuro docente vela por el buen desempeño del futuro ciudadano en una sociedad en la que existen reglas y leyes, de esa manera el docente en formación, mediante la actividad realizada, contribuye a que se determine una convivencia sana y productiva.

El actuar ético corresponde también a la orientación brindada del docente a los alumnos para que puedan desenvolverse en una sociedad de manera plena, también se relaciona con el hecho de brindar una buena enseñanza y educación en valores a partir de situaciones reales con la finalidad de que el alumno sea un individuo preparado y un ciudadano que lleve a ejercer tanto sus derechos como sus obligaciones.

La fortaleza que aquí se desarrolla es la de tomar en cuenta cada una de las materias y asignaturas establecidas en el currículo, asignar un tiempo definido tal como lo establece el plan y programas de estudio destinando un horario particular para su tratamiento, en este caso, hago referencia al estudio de la Formación Cívica y Ética, sin embargo, un área de oportunidad es la

Se identificó que existe congruencia en las 4 fases, puesto que sí corresponde la reflexión a la evidencia mostrada, a los registros de su práctica y a lo escrito en su plan de clase. Como puede observarse en la figura 5, el estudiante normalista logra escribir una reflexión crítica (Lamas, 2016, como se citó en Rodrigues, 2013), pues no solo contiene datos y hechos sobre su experiencia docente, sino que relaciona 
la teoría e identifica su proyección a través de fortalezas, áreas de oportunidad o retos con el fin de transformar su realidad.

\section{DISCUSIÓN}

Es importante la elaboración del portafolio de evidencias de manera metacognitiva por parte de los estudiantes a titularse, porque fomenta la autonomía y el desarrollo del pensamiento reflexivo y crítico; sin embargo, considero que hace falta indagar y profundizar en la obtención de la evidencia de tipo conceptual, procedimental y actitudinal para demostrar en qué medida es logrado el aprendizaje en los niños y en qué grado el estudiante normalista desarrolla la competencia profesional.

\section{CONCLUSIONES}

El grado de confianza que tienen los asesorados con su maestro asesor, desde el primero hasta el octavo semestre, son primordiales para lograr un acercamiento y una profundidad en las preguntas, dudas, elaboración de trabajos y una participación democrática en la elaboración del portafolio de evidencias.

Las fichas de autocorrección, funcionaron como un acompañante metacognitivo, promoviendo en los alumnos la toma de decisiones para seleccionar las competencias profesionales a desarrollar, para seleccionar las evidencias más significativas y para desarrollar ideas en la acción y sobre la acción, incrementando el análisis y la reflexión crítica.

La Secuencia didáctica permite evidenciar el desarrollo de un contenido en varias sesiones, iniciando con la fase de apertura, continuando con la fase de desarrollo y terminando con la fase de cierre; permitiendo vincular el conocimiento previo con el nuevo, estableciendo actividades para propiciar el desarrollo de habilidades y actitudes a lo largo de cada situación didáctica, es decir, promueve el desarrollo de competencias. Como lo menciona (Belmonte y Bernárdez, 2020) "El modelo de formación en competencias implica un proceso de cambio que incide directamente en la visión y pensamiento del estudiante, fomentando el pensamiento crítico y su inteligencia práctica".

La matriz de consistencia permitió observar la congruencia entre el plan de clase, los registros, las evidencias y la reflexión. Además, propició la identificación de fortalezas y áreas de oportunidad del docente en formación, para diseñar un plan de mejora. 


\section{REFERENCIAS}

Abrigo, I. (2018). La matriz de consistencia: una metodología de investigación para desarrollar el estado del arte para emprendimientos artesanales enfocados en las TIC's. https://www.researchgate.net/publication/331357121_La_matriz_de_consistencia_una_metodologia_de _investigacion_para_desarrollar_el_estado_del_arte_para_emprendimientos_artesanales_enfocados_en_ las_TICs

Belmonte, M. y Bernárdez, A. (2020). Perfiles profesionales del pedagogo. https://southfloridapublishing.com/ojs/index.php/jdev/article/view/68/82

Honey, A. (1995). Cuestionario de estilos de aprendizaje. https://antoniortega2000.files.wordpress.com/2014/10/cuestionario-de-estilos-de-aprendizaje-yexplicacion-de-estilos.pdf

Lamas Pelayo, Jessica Vargas D'Uniam. (2016). Los niveles de reflexión en los portafolios de la Práctica Pre Profesional Docente. REDU: Revista de Docencia Universitaria. https://dialnet.unirioja.es/servlet/articulo?codigo $=5765930$

Latorre, A. (2005). La investigación-acción. Conocer y cambiar la práctica educativa. Editorial Grao. https://www.uv.mx/rmipe/files/2019/07/La-investigacion-accion-conocer-y-cambiar-la-practicaeducativa.pdf

SEP, (2012). Orientaciones académicas para la elaboración del trabajo de titulación. https://www.dgespe.sep.gob.mx/public/documentos_orientadores/orientaciones_academicas_para_el_\% 20trabajo_\%20de\%20_titulacion.pdf

SEP, (2017). Modelo Educativo para la Educación Obligatoria. https://www.gob.mx/cms/uploads/attachment/file/207252/Modelo_Educativo_OK.pdf

SEP, (2020). Perfiles profesionales, criterios e indicadores para docentes, técnicos docentes y personal con funciones de dirección y de supervisión.http://file-system.uscmm.gob.mx/20202021/compilacion/Perfiles, \%20Criterios\%20e\%20Indicadores\%20EB\%202020-2021.pdf

Tobón, S. (2010). Secuencias didácticas: Aprendizaje y Evaluación de Competencias. http://files.ctezona141.webnode.mx/200000004-8ed038fca3/secuencias-didacticastobon-120521222400phpapp02.pdf 